Pacific Journal of Mathematics

TORSIONFREE INJECTIVE MODULES 


\title{
TORSIONFREE INJECTIVE MODULES
}

\author{
MARK L. TEPLY
}

The usual torsion theory for modules over an integral domain has the following well-known property:

(A) Any direct sum of torsionfree injective modules is injective.

This property does not always hold for torsion theories over a more general ring $R$. The main theorem of this paper determines nine necessary and sufficient conditions for (A) to hold in the setting of more general torsion theories. In the case that every module is considered to be torsionfree, then the conditions in the main theorem reduce to well-known conditions for the ring $R$ to be left Noetherian. If a hereditary torsion theory satisfies (A), then its associated torsion filter possesses a cofinal subset of finitely generated left ideals. As applications of the main theorem, the torsionfree covers of Enochs are generalized to more general notions of torsion over more general rings, and then it is shown that the class of $R$ modules for which the torsionfree quotient with respect to the Goldie torsion theory is injective forms a torsion theory if and only if property (A) holds for the Goldie torsion theory of $R$-modules.

In this paper all rings $R$ are assumed to possess a unit element 1 , and all $R$-modules are left unitary modules.

Before proceeding, we review from [3] the definition and properties of a torsion theory for $R$-modules: A torsion theory $(\mathscr{T}, \mathscr{F}$ ) consists of a pair of classes of $R$-modules satisfying the following axioms:

(1) $\mathscr{T} \cap \mathscr{F}=0$.

(2) If $T \rightarrow A \rightarrow 0$ is exact and $T \in \mathscr{T}$, then $A \in \mathscr{T}$.

(3) If $0 \rightarrow A \rightarrow F$ is exact and $F \in \mathscr{F}$, then $A \in \mathscr{F}$.

(4) For each $R$-module $X$ there exists an exact sequence $0 \rightarrow$ $T \rightarrow X \rightarrow F \rightarrow 0$ with $T \in \mathscr{T}$ and $F \in \mathscr{F}$.

$\mathscr{T}$ is called the torsion class, and $\mathscr{F}$ is called the torsionfree class. The torsion class $\mathscr{T}$ is closed under homomorphic images, direct sums, and extensions, i.e., $0 \rightarrow A \rightarrow X \rightarrow B \rightarrow 0$ exact and $A, B \in \mathscr{T}$ imply $X \in \mathscr{T}$. The torsionfree class $\mathscr{F}$ is closed under submodules, direct products, and extensions.

A torsion theory $(\mathscr{T}, \mathscr{F})$ is called hereditary if it has the following property: If $0 \rightarrow A \rightarrow T$ exact and $T \in \mathscr{T}$, then $A \in \mathscr{T} \cdot$ A torsion theory is hereditary if and only if $\mathscr{F}$ is closed under injective envelopes, i.e., $F \in \mathscr{F}$ implies $E(F) \in \mathscr{F}$. With each hereditary torsion theory, there is an associated topologizing and idempotent filter of 
left ideals: $F(\mathscr{S})=\{L \mid L$ is a left ideal of $R, R / L \in \mathscr{T}\}$. We shall consistently use $F(\mathscr{T})$ to denote the filter associated with $\mathscr{T}$. For a hereditary torsion theory, $(\mathscr{T}, \mathscr{F}), R x \in \mathscr{T}$ if and only if $I x=0$ for some $I \in F(\mathscr{T})$. $\mathscr{T}(M)$ denotes the torsion submodule of $M \in_{R} \mathbb{l l}$.

1. Any direct sum of torsionfree injectives is injective. For brevity in the sequel, we call a homomorphism $\phi: A \rightarrow \oplus \sum_{\alpha \in w} F_{\alpha}$ a finite homomorphism $(F H)$ if $\phi(A)$ has nonzero coordinates in only finitely many $\left\{F_{\alpha}\right\}_{\alpha \in w}$.

Now we prove a technical lemma:

Lemma 1.1. Let $(\mathscr{F}, \mathscr{F})$ be a hereditary torsion theory. Let $\mathscr{T}(R) \subseteq I_{1} \subset I_{2} \subset I_{3} \subset \cdots$ be a countable infinite ascending chain of left ideals of $R$, let $I=\bigcup_{i=1}^{\infty} I_{i}$, let $\left\{F_{\alpha}\right\}_{\alpha \in w}$ be a collection of torsionfree injectives, and let $\phi: I \rightarrow \bigoplus \sum_{\alpha \in w} F_{\alpha}$ be a homomorphism such that $\dot{\phi} \mid I_{i}$ is $(F H) \forall i$. If there exists $I_{k}$ such that $I / I_{k} \in \mathscr{T}$, then $\dot{\phi}$ can be extended to a homomorphism $f: R \rightarrow \oplus \sum_{\alpha \in w} F_{\alpha}$ and $f$ is $(F H)$.

Proof. Consider the diagram:

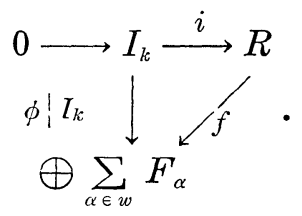

Since $\dot{\phi} \mid I_{k}$ is $(F H)$, there exists $f$ making (B) commute. Since both $\dot{\phi}$ and $f \mid 1$ extend $\dot{\phi} \mid I_{k}$, then $I_{k} \subseteq \operatorname{ker}(\dot{\phi}-f \mid I)$. Hence $\dot{\phi}-f \mid I$ induces a homomorphism $\theta: I / I_{l} \rightarrow \bigoplus \sum_{\alpha \in W} F_{\alpha}$. Since $I / I_{k} \in \mathscr{T}$ and $\oplus \sum_{\alpha \in W} F_{\alpha} \in \mathscr{F}$, then $\theta=0$ and $\phi=f \mid I$.

Given a hereditary torsion theory $(\mathscr{F}, \mathscr{F})$, we call a left ideal of $R$ an $\mathscr{F}$-ideal if it is the order ideal of a generator of a torsionfree cyclic $R$-module, i.e., $L$ is a $\mathscr{F}$-ideal if and only if $L \in\{K \mid R /$ $K \in \mathscr{F}\}$ if and only if $x \in R-L$ implies $(L: x) \notin F(\mathscr{T})$. Given a set of $\mathscr{F}$-ideals we can determine the filter $F(\mathscr{T})$ to be $\{I / I$ is a left ideal of $R ; I \leqq J$ and $J$ is $\mathscr{F}$-ideal $\Longrightarrow J=R\}$. Conversely, given $F(\mathscr{T})$ we can determine the $\mathscr{F}$-ideals to be

$$
R \cup\{I \mid(I: r) \notin F(\mathscr{T}) \forall r \in R\} .
$$

Note that $\mathscr{F}$ is closed under homomorphic images if and only if the following property holds: $I$ is an $\mathscr{F}$-ideal and $I \subseteq K$ implies $K$ is an $F$-ideal.

We now come to our main theorem:

THEOREM 1.2. Let $(\mathscr{T}, \mathscr{F})$ be a hereditary torsion theory. 
Then the following are equivalent:

(1) Any direct sum of torsionfree injective modules is injective.

(2) Any direct sum of countably many torsionfree injective modules is injective.

(3) $R$ has acc on $\mathscr{F}$-ideals.

(4) If $I_{1} \subset I_{2} \subset I_{3} \subset \cdots$ is a strictly increasing countably infinite chain of left ideals of $R$, then there exists an integer $r$ such that $\left[\bigcup_{i=1}^{\infty} I_{i}\right] / I_{r} \in \mathscr{T}$.

(5) If $\mathscr{T}(R) \subset I_{1} \subset I_{2} \subset I_{3} \subset \cdots$ is a strictly increasing countably infinite chain of left ideals of $R$, then there exists an integer $r$ such that $\left[\bigcup_{i=1}^{\infty} I_{i}\right] / I_{r} \in \mathscr{T}$.

(6) Every torsionfree injective module is the direct sum of indecomposable modules.

(7) There exists a cardinal number $c$ such that every injective element of $\mathscr{F}$ is a direct sum of modules each of which is generated by $c$ elements.

(8) There exists a cardinal number $d$ such that every injective element of $\mathscr{F}$ is a direct sum of injective envelopes of modules each of which is generated by $d$ elements.

(9) There exists a cardinal number $c$ such that each injective module in $\mathscr{F}$ is contained in a direct sum of modules each of which is generated by $c$ elements.

REmark. In case $\mathscr{T}=\{0\}$, then every left ideal of $R$ is an $\mathscr{F}$-ideal. Hence as a special case of Theorem 1.2 we get the following for $\mathscr{T}=\{0\}$ :

$(1) \Leftrightarrow(2) \Leftrightarrow(3)$ is [5] Theorem 0.1 (Bass, Cartan, Eilenberg, Papp).

$(1) \Rightarrow(6)$ is [5] Theorem 0.3 (Matlis, Papp).

$(6) \Leftarrow(1)$ is [5] Corollary 1.3 (Papp).

$(1) \Leftrightarrow(7)$ is [5] Theorem 1.1.

$(1) \Leftrightarrow(8)$ is [5] Corollary 1.2.

$(1) \Leftrightarrow(9)$ is [5] Theorem 3.1.

Proof of Theorem 1.2. (1) $\Rightarrow(2)$ : Trivial.

(2) $\Rightarrow(3)$ : Let $I_{1} \subset I_{2} \subset I_{3} \subset \cdots$ be a countably infinite strictly ascending chain of $\mathscr{F}$-ideals. Then $I_{i}=\left(0: x_{i}\right)$ for some $R x_{i} \in \mathscr{F}$, and hence $E\left(R x_{i}\right) \in \mathscr{F}$. Set $I=\bigcup_{i=1}^{\infty} I_{i}$ and $F=\bigoplus \sum_{i=1}^{\infty} E\left(R x_{i}\right)$. Note that $I x_{i} \neq 0$ for all $i$. Define $f: I \rightarrow F$ via $f(y)=\left(y x_{i}\right)$ for all $y \in I$. By (2) there exists $z \in F$ such that $f(y)=y z$ for all $y \in I$. But $z$ is nonzero in only finitely many coordinants of $F$, and hence $f(I)$ is nonzero in only finitely many coordinants of $F$. This contradicts $I x_{i} \neq 0$ by the definition of $f$. Thus $R$ must have acc on $\mathscr{F}$-ideals.

$(3) \Rightarrow(4)$ : Let $I_{1} \subset I_{2} \subset I_{3} \subset \cdots$ be an infinite ascending chain. Define $K_{i}$ by $K_{i} / I_{i}=\mathscr{T}\left(R / I_{i}\right)$. Then $R / K_{i} \cong\left(R / I_{i}\right) /\left(K_{i} / I_{i}\right) \in \mathscr{F}$, and 
$K_{1} \subseteq K_{2} \subseteq K_{3} \subseteq \cdots$. By (3) there exists an integer $r$ such that $K_{r}=K_{r+1}=\cdots$. Thus $\bigcup_{i=1}^{\infty} I_{i} \leqq \bigcup_{i=1}^{\infty} K_{i}=K_{r}$. Hence $\left[\bigcup_{i=1}^{\infty} I_{i}\right] / I_{r} \leqq$ $K_{r} / I_{r} \in \mathscr{T}$.

$(4) \Rightarrow(5)$ : Trivial.

$(5) \Rightarrow(1)$ : Let $I$ be a left ideal, $\left\{F_{\alpha}\right\}_{\alpha \in W}$ a set of torsionfree injectives, and $\dot{\phi}: I \rightarrow \oplus \sum_{\alpha \in W} F_{\alpha}$. It is sufficient to show that $\phi$ can be extended to a homomorphism from $R$ to $\oplus \sum_{\alpha \in W} F_{\alpha}$. Since $\oplus \sum_{\alpha \in W} F_{\alpha} \in \mathscr{F}$, then $\phi \mid \mathscr{T}(I)=0$, so we can extend to $I+\mathscr{T}(R)$ by defining the extension map to be 0 on $\mathscr{T}(R)$. Furthermore, any extension of $\phi$ to $R$, say $f: R \rightarrow \oplus \sum_{\alpha \in W} F_{\alpha}$, has the property that $f \mid \mathscr{T}^{-}(R)=0$. Thus we may assume $\mathscr{T}^{-}(R) \subseteq I$.

If $I / \mathscr{G}(R)$ is finitely generated, then $\phi$ is $(F H)$ and hence we can extend $\dot{\phi}$ as desired. If $I / \mathscr{T}(R)$ is not finitely generated, then choose $x_{i} \in I(i=1,2,3, \cdots)$ such that $\mathscr{T}(R) \subset \mathscr{T}(R)+\left(x_{1} \mid \subset \mathscr{T}(R)+\right.$ $\left(x_{1}, x_{2} \mid \subset \cdots\right.$. Set $J_{1}=\mathscr{T}(R)+\bigcup_{n=1}^{\infty}\left(x_{1}, x_{2}, \cdots, x_{n} \mid\right.$. If $I=J_{1}$, then we are done by (5) and Lemma 1.1. If $I \supset J_{1}$, we proceed by (transfinite) induction.

Suppose $\beta$ is not a limit ordinal.

Case 1. $I / J_{\beta-1}$ is finitely generated. Set $I_{\beta}=I$. By induction hypothesis there exists $(F H) f_{\beta-1}: R \rightarrow \bigoplus \sum_{\alpha \in W} F_{\alpha}$ such that $f_{\beta-1} \mid J_{\beta-1}=$ $\phi \mid J_{\beta-1}$. Since $I / J_{\beta-1}$ is finitely generated, it follows that $\phi \mid I$ is $(F H)$, and hence we can extend $\dot{\phi}$ as desired.

Case 2. $I / J_{\beta-1}$ is not finitely generated. Choose $x_{\beta i} \in I(i=1,2$, $3, \cdots)$ such that $J_{\beta-1} \subset J_{\beta-1}+\left(x_{\beta 1} \mid \subset J_{\beta-1}+\left(x_{\beta 1}, x_{\beta 2} \mid \subset \cdots\right.\right.$. Set $J_{\beta}=$ $J_{\beta-1}+\bigcup_{n=1}^{\infty}\left(x_{\beta 1}, x_{\beta 2}, \cdots, x_{\beta n} \mid\right.$. By (5), the induction hypothesis, and Lemma 1.1, it follows that $\phi \mid J_{\beta}$ is $(F H)$.

If $\beta$ is a limit ordinal, then let $J_{\beta}=\sum_{r<\beta} J_{\gamma}$. Suppose $\phi \mid J_{\beta}$ is not $(F H)$. Then there exists a sequence $\left\{\gamma_{i}\right\}$ such that

(i) $\gamma_{i}<\beta \forall i$

(ii) $i<j$ implies $\gamma_{i}<\gamma_{j}$

(iii) $\dot{\phi}\left(J_{\gamma i}\right)$ has nonzero coordinates in at least $i$ of the $\left\{F_{\alpha}\right\}_{\alpha \in \eta}$.

If $\bigcup_{i=1}^{\infty} J_{\gamma_{i}} \subset J_{\beta}$ (properly), then there exists $\sigma<\beta$ such that $\bigcup_{i=1}^{\infty} J_{\gamma_{i}} \subseteq J_{o}$. Hence $\phi \mid J_{\sigma}$ is not $(F H)$, a contradiction to our induction hypothesis. Hence $\bigcup_{i=1}^{\infty} J_{r_{i}}=J_{\beta}$. So using (5) and Lemma 1.1, it follows that $\dot{\phi} \mid J_{\beta}$ is $(F H)$, a contradiction to our assumption. Thus $\phi \mid J_{\beta}$ is $(F H)$ as desired.

By induction it follows that $\phi \mid I$ is $(F H)$, and hence $\phi$ can be extended as desired.

$(1) \Rightarrow(6)$ : Any torsionfree injective $R$-module $M$ has the following property whenever (1) holds:

(C) Every union of an ascending chain of direct summands of $M$ is a direct summand of $M$. 
To see this, we argue by transfinite induction: Let $\left\{M_{\alpha}\right\}_{\alpha \in I}$ be an ascending chain of direct summands of $M$ whose union is $N$. Choose $x_{1} \in N$. Then $x_{1} \in M_{\alpha}$ for some $\alpha \in I$. But $M_{\alpha}$ is a summand of $M$, and hence $M_{\alpha}$ is injective. It follows that $E\left(R x_{1}\right) \cong M_{\alpha}$. Suppose $\gamma$ is not a limit ordinal, and suppose that $E\left(R x_{\beta}\right) \subseteq N$ have been constructed for $\beta<\gamma$ such that $\sum_{\beta \leqq \gamma-1} E\left(R x_{\beta}\right)$ is direct. From (1) it follows that $\sum_{\beta \leqq r-1} E\left(R x_{\beta}\right)$ is injective and hence is a summand of $N$. Write $N=\sum_{\beta \leqq r-1} E\left(R x_{\beta}\right) \oplus K$, and choose $x_{\gamma} \in K$. Then $x_{\gamma} \in M_{\alpha}$ for some $\alpha$, so again $E\left(R x_{\gamma}\right) \subseteq M_{\alpha} \leqq N$; moreover, $\sum_{\beta \leqq \gamma} E\left(R x_{\beta}\right)$ is direct.

If $\gamma$ is a limit ordinal, consider $\sum_{\beta<\gamma} E\left(R x_{\beta}\right)$, where $\sum_{\beta<\sigma} E\left(R x_{\beta}\right)$ is direct for any $\sigma<\gamma$. If $x \in E\left(R x_{i}\right) \cap \sum_{\beta<\gamma, \beta \neq \delta} E\left(R x_{\beta}\right)$, then

$$
x \in E\left(R x_{\delta}\right) \cap \sum_{\beta \in F} E\left(R x_{\beta}\right)
$$

where $F$ is a finite subset of $I$ such that $\beta \in F$ implies that $\beta<\gamma$. Then $x=0$, and hence $\sum_{\beta<\gamma} E\left(R x_{\beta}\right)$ is direct. By $(1), \oplus \sum_{\beta<\gamma} E\left(R x_{\beta}\right)$ must be an injective submodule of $N$, and hence $E\left(R x_{\gamma}\right)$ can be constructed in the complementary summand of $\oplus \sum_{\beta<\gamma} E\left(R x_{\beta}\right)$ as before.

By transfinite induction it follows that $N=\bigoplus \sum_{\gamma<\beta} E\left(R x_{\gamma}\right)$ for some ordinal $\beta$. Then by (1) $N$ is injective and hence a summand of $M$.

Using property $(\mathrm{C})$, the proof of $(1) \Rightarrow(6)$ is an easy modification of [8] Theorem 1.

$(6) \Rightarrow(1)$ : This follows by an easy modification of the proof of [8] Theorem 2.

$(6) \Rightarrow(7) \Rightarrow(1)$ : Since $\mathscr{F}$ is closed under products and submodules, this follows by an easy modification of the proof of [5] Theorem 1.1.

$(7) \Rightarrow(8)$ : Trivial.

$(8) \Rightarrow(7)$ : By $(8)$, each injective module in $\mathscr{F}$ is a direct sum of injective envelopes of homomorphic images of modules

$$
\text { (*) } \bigoplus \sum_{\alpha \in W} R / I_{\alpha}
$$

where $I_{\alpha}$ is an $\mathscr{F}$-ideal and $|w|=d$. Since there is only a set of $\mathscr{F}$-ideals, then there is only a set of modules of the form $\left({ }^{*}\right)$. Since each module of the form $\left(^{*}\right)$ has only a set of submodules, then there is only a set of nonisomorphic homomorphic images of modules of the form $\left(^{*}\right)$. Let $\left\{K_{\beta} \mid \beta \in \mathscr{B}\right\}$ denote this set of isomorphism classes of homomorphic images. Then each $E\left(K_{\beta}\right)$ is generated by $c=\sum_{\beta \in \mathscr{O}}\left|E\left(K_{\beta}\right)\right|$ elements.

$(7) \Leftrightarrow(9)$ : This follows by an easy modification of the proof of [5] Theorem 3.3.

CoRollary 1.3. Let $\left(\mathscr{T}^{-}, \mathscr{F}\right)$ be a hereditary torsion theory. 
If $R / \mathscr{T}(R)$ is a left Noetherian ring, then any direct sum of torsionfree injective modules is injective.

F. Anderson has pointed out (oral communication) that the proof of $(2) \Rightarrow(3)$, which is a modification of an argument of Bass, can be easily modified to give the following result:

Corollary 1.4. Let I be a class of injective $R$-modules. If any direct sum of modules in $I$ is injective, then $R$ has acc on the annihilators of modules in $I$. Let $J$ be a class of $R$-modules with the property that $M \in J$ implies $E(M) \in J$. If any direct sum of injective modules in $J$ is injective, then $R$ has acc on the annihilators of modules in $J$.

A filter $F(\mathscr{T})$ of left ideals for a hereditary torsion theory ( $\mathscr{T}$, $\mathscr{F})$ is said to have a cofinal subset of finitely generated left ideals if, for each $I \in F(\mathscr{T})$, there exists a finitely generated left ideal $J \subseteq I$ such that $J \in F(\mathscr{T})$.

Theorem 1.5. Let $(\mathscr{S}, \mathscr{F})$ be a hereditary torsion theory. If the direct sum of torsionfree injectives is injective, then $F(\mathscr{T})$ has a cofinal subset of finitely generated left ideals.

Proof. Let $I \in F(\mathscr{T})$ with $I$ not finitely generated. By transfinite induction we shall construct a strictly increasing chain $\left\{I_{\alpha}\right\}_{\alpha \in W}$ of left ideals contained in $I$, each of which possesses of finitely generated left ideal $J_{\alpha}$ such that $I_{\alpha} / J_{\alpha} \in \mathscr{T}$.

Choose $x_{1 i} \in I(i=1,2, \cdots)$ such that $\left(x_{11} \mid \subset\left(x_{11}, x_{12} \mid \subset\left(x_{11}, x_{12}\right.\right.\right.$, $x_{13} \mid \subset \cdots$. Set $I_{1}=\bigcup_{n=1}^{\infty}\left(x_{11}, \cdots, x_{1 n} \mid\right.$. By Theorem 1.2 (4) there exists $J_{1}=\left(x_{11}, \cdots, x_{1 m}\right)$ such that $I_{1} / J_{1} \in \mathscr{S}$. If $I_{1}=I$, we are done. If not, construct as follows:

Suppose $\alpha$ is not a limit ordinal. We can consider two cases:

Case 1. $I / I_{\alpha-1}$ is not finitely generated. Choose $x_{\alpha i} \in I(i=1,2, \cdots)$ such that $I_{\alpha-1} \subset I_{\alpha-1}+\left(x_{\alpha 1} \mid \subset I_{\alpha-1}+\left(x_{\alpha 1}, x_{\alpha 2} \mid \subset \cdots\right.\right.$. Set

$$
I_{\alpha}=\bigcup_{n=1}^{\infty}\left(x_{\alpha 1}, \cdots, x_{\alpha n} \mid+I_{\alpha-1} .\right.
$$

By Theorem 1.2 (4) there exists $K=\left(x_{\alpha 1} \cdots, x_{\alpha m}\right)$ for some $m$ such that $I_{\alpha} / K+I_{\alpha-1} \in \mathscr{T}$. By the induction hypothesis there exists a finitely generated left ideal $J_{\alpha-1}$ such that $I_{\alpha-1} / J_{\alpha-1} \in \mathscr{S}^{-}$. Then observe that

$$
\frac{I_{\alpha-1}+K}{J_{\alpha-1}+K}=I_{\alpha-1} / J_{\alpha-1}+\left(K \cap I_{\alpha-1}\right)
$$

is a homomorphic image of $I_{\alpha-1} / J_{\alpha-1} \in \mathscr{T}$. Hence 


$$
\frac{I_{\alpha-1}+K}{J_{\alpha-1}+K} \in \mathscr{S} \text {. }
$$

So from the exact sequence

$$
0 \rightarrow \frac{I_{\alpha-1}+K}{J_{\alpha-1}+K} \rightarrow \frac{I_{\alpha}}{J_{\alpha-1}+K} \rightarrow \frac{I_{\alpha}}{I_{\alpha-1}+K} \rightarrow 0
$$

it follows that $I_{\alpha} / J_{\alpha-1}+K \in \mathscr{T}$. Set $J_{\alpha}=J_{\alpha-1}+K$. Since $J_{\alpha}$ is finitely generated as a left ideal, we have the desired $I_{\alpha}$ and $J_{\alpha}$.

Case 2. $I / I_{\alpha-1}$ is finitely generated, say by $\left\{y_{i}+I_{\alpha-1} \mid i=1,2,3\right.$, $\cdots, N\}$. Set $I_{\alpha}=I$ and $J_{\alpha}=J_{\alpha-1}+\left(y_{1}, y_{2}, \cdots, y_{N} \mid\right.$. Then $J_{\alpha}$ has the properties: $J_{\alpha}$ is finitely generated and $I_{\alpha} / J_{\alpha} \in \mathscr{T}$.

Suppose $\alpha$ is a limit ordinal. Then let $I_{\alpha}=\sum_{\gamma<\alpha} I_{r}$. Define $K_{\gamma}$ by $K_{\gamma} / I_{\gamma}=\mathscr{T}\left(R / I_{\gamma}\right)$. Then $\left\{K_{r}\right\}$ is a set of $\mathscr{F}$-ideals. Let $\gamma_{1}$ be the first ordinal $\leqq \alpha$ (if it exists) such that $I_{\gamma_{1}} \nsubseteq K_{1}$. Let $\gamma_{2}$ be the first limit ordinal $\leqq \alpha$ such that $I_{\gamma_{2}} \nsubseteq K_{\gamma_{1}}$. Proceeding inductively we obtain a chain $K_{1} \subset K_{\gamma_{1}} \subset K_{\gamma_{2}} \subset \ldots$. By Theorem 1.2 (3) $R$ has acc on $\mathscr{F}$-ideals, and hence the chain must stop, say at $K_{\beta}\left(\beta=\gamma_{m}\right.$ for some $m$.) It follows that $I_{\alpha} \subseteq K_{\beta}$; and hence $I_{\alpha} / I_{\beta} \in \mathscr{T}$, since $\mathscr{T}$ closed under submodules. By induction hypothesis there exists $J_{\beta}$ such that $J_{\beta}$ is finitely generated and $I_{\beta} / J_{\beta} \in \mathscr{T}$. Thus the exact sequence

$$
0 \rightarrow \frac{I_{\beta}}{J_{\beta}} \rightarrow \frac{I_{\alpha}}{J_{\beta}} \rightarrow \frac{I_{\alpha}}{I_{\beta}} \rightarrow 0
$$

implies $I_{\alpha} / J_{\beta} \in \mathscr{T}$. Setting $J_{\alpha}=J_{\beta}$, we have our construction for the limit ordinal case.

By induction it follows that $I=I_{\beta}$ for some ordinal $\beta$. Thus the exact sequence

$$
0 \rightarrow I / J_{\beta} \rightarrow R / J_{\beta} \rightarrow R / I \rightarrow 0
$$

implies $R / J_{\beta} \in \mathscr{T}$, and hence $J_{\beta} \in F(\mathscr{S})$.

To see that the converse of Theorem 1.5 does not hold, we give the following example: Let $I$ be a non-Noetherian ring and $R=K+I$ (ring direct sum). As in [6] we can construct $(\mathscr{T}, \mathscr{F})$ via $\mathscr{T}=$ $\{M \mid I M=0\}$ where $I=\bigcap_{L \in F(\mathscr{T})} L$. Then $F(\mathscr{T})$ has a cofinal subset of finitely generated left ideals (we can always use $I$ which is generated by the unit element of $I$ ), but the direct sum of torsionfree injectives will not always be injective.

For the case that $\mathscr{F}$ is also $T T F$ class studied in [6] (i.e., $\mathscr{F}$ is closed under homomorphic images) we obtain:

Proposition 1.6. Let $(\mathscr{T}, \mathscr{F})$ be a hereditary torsion theory 
such that $\mathscr{F}$ is closed under homomorphic images. Then the following are equivalent:

(1) Any direct sum of torsionfree injectives is injective.

(2) $R / \mathscr{T}(R)$ is a left Noetherian ring.

In the case that a hereditary torsion theory has $\mathscr{T}$ closed under injective envelopes and $\mathscr{F}$ closed under homomorphic images, rings for which any direct sum of torsionfree injectives is injective take a particulary nice form. This will be a consequence of Proposition 1.6 and the following proposition which is of interest in its own right:

Proposition 1.7. If $(\mathscr{T}, \mathscr{F})$ is a hereditary torsion theory such that $\mathscr{T}$ is closed under injective envelopes and $\mathscr{F}$ is closed under homomorphic images, then $\mathscr{T}(M)$ is a direct summand of $M \forall M \in_{R} \mathscr{C l}$.

Proof. Let $M \in_{R} \mathscr{C l}$. If $M$ is an essential extension of $\mathscr{T}(M)$, then $M \in \mathscr{T}$; and hence $M$ has $\mathscr{T}(M)$ as a trivial direct summand. If $M$ is not an essential extension of $\mathscr{T}(M)$, then there exists a nonzero submodule $N$ of $M$ maximal in the property that $N \cap \mathscr{T}(M)=0$. Clearly $N \oplus \mathscr{T}(M) \leqq M . \quad M / N+\mathscr{T}(M)$ is a homomorphic image of $M / \mathscr{T}(M)$ and hence is in $\mathscr{F}$. But $M / N$ is an essential extension of $\mathscr{T}(M)+N / N \cong \mathscr{T}(M) \in \mathscr{T}$, and hence $M / N \in \mathscr{T}$. Since $\mathscr{T}$ closed under homomorphic images, it follows that

$$
M / N+\mathscr{T}(M) \in \mathscr{T} \cap \mathscr{F}=0 .
$$

Therefore $M=\mathscr{T}(M) \oplus N$ are desired.

CoROllary 1.8. Let $(\mathscr{T}, \mathscr{F})$ be a hereditary torsion theory such that $\mathscr{F}$ is closed under homomorphic images and $\mathscr{T}$ is closed under injective envelopes. Then the following are equivalent:

(1) Any direct sum of torsionfree injective modules is injective.

(2) $R=\mathscr{T}(R)+N$ (ring direct sum) where $N$ is a left Noetherian ring.

2. Torsionfree covers. In this section all torsion theories considered will be hereditary.

To generalize the notion of torsionfree covers of [4], we need a definition of "purity" for $R$-modules such that pure submodules have the following property: If $F \in \mathscr{F}$, then $N$ is a pure submodule of $F$ if and only if $F / N \in \mathscr{F}$. So select any definition of purity for which this property holds. Such definitions exist in the literature; for example, see [7] Proposition 6.2.

Proposition 2.1. Let $F \in \mathscr{F}$. Then: 
(1) $N$ is pure in $F$ if and only if $F / N \in \mathscr{F}$.

(2) If $A \subseteq B \subseteq F, A$ pure in $F$, and $B / A$ pure in $F / A$, then $B$ is pure in $F$.

(3) If $F(\mathscr{G})$ has a cofinal subset of finitely generated left ideals, then the union of a chain of pure submodules of $F$ is pure in $F$.

\section{Proof.}

(1) By definition of pure.

(2) Apply (1).

(3) By (1) it follows that $N$ is pure in $F \in \mathscr{F}$ if and only if the following property holds: If $x \in F-N$ and $I \in F(\mathscr{T})$, then $I x \nsubseteq N$. Suppose $\left\{F_{\alpha}\right\}_{\alpha \in W}$ is a chain of pure submodules of $F$, but $\bigcup_{\alpha \in W} F_{\alpha}$ is not pure in $F$. Then there exists $x \in F-\bigcup_{\alpha \in W} F_{\alpha}$ and $I \in F(\mathscr{T})$ such that $I x \subseteq \bigcup_{\alpha \in W} F_{\alpha}$. Since $F(\mathscr{T})$ has a cofinal subset of finitely generated left ideals, then there exists $J \subseteq I$ such that $J \in F(\mathscr{G})$ and $J$ is finitely generated. But then $J x \subseteq \bigcup_{\alpha \in W} F_{\alpha}$ and $J$ finitely generated implies there exists $\beta \in W$ such that $J \rightsquigarrow \subseteq F_{\beta}$, contradicting the purity of $F_{\beta}$ in $F$.

We now define a torsionfree covering of an $R$-module $M$ as an $R$-module $T(M) \in \mathscr{F}$ together with an epimorphism is:T(M) $\rightarrow M$ such that

(*) ker $\psi$ contains no pure submodules of $T(M)$

(**) For each homomorphism $\phi: F \rightarrow M$ with $F \in \mathscr{F}$ there exists $f: F \rightarrow T(M)$ such that $\psi \circ f=\dot{\phi}$.

Using the fact that $\mathscr{T}$ closed under submodules implies $\mathscr{F}$ is closed under injective envelopes, we have the following lemma by the same methods of proof used by Enochs [4]:

\section{Lemma 2.2. Let $R \in \mathscr{F}$. Then:}

(1) If $\psi: M^{\prime} \rightarrow M$ satisfies (**) and $N \subseteq M$, then

$$
\dot{\psi} \mid \psi^{-1}(N): \psi^{-1}(N) \rightarrow N
$$

satisfies $\left({ }^{* *}\right)$.

(2) If $M$ is injective, then $\psi: M^{\prime} \rightarrow M$ satisfies (**) if and only if, for every homomorphism $\phi: F \rightarrow M$ with $F \in \mathscr{F}$ and $F$ injective, there exists $f: F \rightarrow M^{\prime}$ such that $\psi \circ \circ=\dot{\phi}$.

(3) If $\psi: M^{\prime} \rightarrow M$ satisfies $\left(^{* *}\right), N$ pure in $M^{\prime}$, and $N \leqq \operatorname{ker} \psi$, then $\bar{\psi}: M^{\prime} / N \rightarrow M$ satisfies $\left.{ }^{* *}\right)$.

Lemma 2.3. Suppose that any direct sum of torsionfree injectives is injective, and let $R \in \mathscr{F}$. Then for every $R$-module $M$, there exists a epimorphism $\psi$ : $M^{\prime} \rightarrow M$ satisfying (**). 
Proof. By Lemma 2.2 (1), we may assume $M$ is injective; so by Lemma 2.2 (2), we may assume $F \in \mathscr{F}$ is injective where $\phi: F \rightarrow M$. $\left\{K_{\alpha} \mid \alpha \in w\right\}$ donotes a set of representatives of the isomorphism classes of injective hulls of cyclic $R$-modules in $\mathscr{F}$. Since any direct sum of torsionfree injectives is injective, it follows that $F=\oplus \sum_{\alpha \in 乃} K_{\alpha}$ where $\mathscr{B}$ is an appropriate index set. Let $I(\alpha)=\operatorname{Hom}\left(K_{\alpha}, M\right)$, and let $K_{\alpha \sigma}$ be a copy of $K_{\alpha}$ for each $\sigma \in I(\alpha)$. Define $M^{\prime}=\bigoplus \sum_{\alpha \in W} \sum_{\sigma \in I(\alpha)} K_{\alpha \sigma}$, and define $\psi: M^{\prime} \rightarrow M$ via $\psi \mid K_{\alpha \sigma}=\sigma$. Then we can define $f: F \rightarrow M^{\prime}$ in the obvious way such that $\psi \circ f=\dot{\phi}$. Hence $\left({ }^{* *}\right)$ is satisfied. Since $R \in \mathscr{F}, \psi$ is an epimorphism.

THEOREM 2.4. Let $(\mathscr{T}, \mathscr{F})$ be a hereditary torsion theory with the following properties:

(1) $R \in \mathscr{F}$.

(2) Any direct sum of torsionfree injective modules is injective. Then every $R$-module has a unique torsionfree covering.

Proof. Using the results developed in this section, the existence part of the proof proceeds as in [4] Theorem 1. Uniqueness: Suppose $\psi^{\prime}: M^{\prime} \rightarrow M$ and $\psi^{\prime \prime}: M^{\prime \prime} \rightarrow M$ are torsionfree covers of $M$. Then there exists $f: M^{\prime} \rightarrow M^{\prime \prime}$ with $\operatorname{Ker} f=K$ and $\psi^{\prime}=\psi^{\prime \prime} \circ f$. Clearly $K \cong \operatorname{ker} \psi^{\prime}$. Let $x \in M^{\prime}-K$. If $I x \leqq K$ for some $I \in F(\mathscr{T})$, then $0=f(I x)=I f(x)$. But then $M^{\prime \prime} \in \mathscr{F}$ and $I \in F(\mathscr{T})$ implies $f(x)=0$ and hence $x \in K$, a contradiction. Thus $I x \nsubseteq K$, and hence $K$ is pure in $M^{\prime}$. Since $M^{\prime}$ is a torsionfree cover, then $K=0$. Thus $f$ is an isomorphism and Card $\left(M^{\prime}\right) \leqq$ Card $\left(M^{\prime \prime}\right)$. The rest of the proof follows by a Zorn's lemma argument as in [4] Theorem 2.

REMARKs. (i) The hypotheses of Theorem 2.4 are satisfied by the usual torsion theory for modules over an integral domain.

(ii) Hypothesis (2) of Theorem 2.4 is satisfied for every hereditary torsion theory of modules over a left Noetherian ring. Thus, for $R$ left Noetherian, Theorem 2.4 holds for the $E(R)$-torsion theory, i.e., the smallest torsion theory for which $E(R)$ is torsionfree (see [6]). Also for left Noetherian rings with zero socle, Theorem 2.4 holds for the simple torsion theory of Dickson, i.e., the smallest torsion theory for which all simple modules are torsion (see [3]).

(iii) In order to insure that $\psi$ is epic in Theorem 2.4, it is necessary to impose a hypothesis such as (1). For if $R$ has left dcc, then the simple torsion theory [3] satisfies all the hypotheses of Theorem 2.4 except (1), but 0 is the only module which as a torsionfree covering. Also the Goldie torsion theory, $(\mathscr{D}, \mathscr{F})$, i.e., the torsion theory whose torsion class is generated by all factor modules 
$B / A$ with $B$ an essential extension of $A$ (see [1]), for modules over a ring $R=\mathscr{D}(R)+S$ (ring direct sum) where $S$ is semisimple with minimum condition satisfies all the hypotheses of Theorem 2.4 except (1), but the class of $R$-modules possessing a torsionfree cover coincides with $\mathscr{F}$.

(iv) For another generalization of Enoch's work using Utumi's rings of quotients, see [2].

3. Goldie torsionfree injectives. The Goldie torsion theory, $(\mathscr{D}, \mathscr{F})$ is the torsion theory whose torsion clas $\mathscr{D}$ is generated by all factor $R$-modules $B / A$ with $B$ an essential extension of $A$ (see [1]). The torsion class $\mathscr{D}$ is closed under submodules and injective envelopes, and hence $\mathscr{F}$ is also closed under injective envelopes. In [1] Alin and Dickson show (Theorem 3.1) that $A / \mathscr{D}(A)$ is injective for all $R$-modules $A$ if and only if $R=\mathscr{D}(R)+S$ (ring direct sum) where $S$ is semisimple with minimum condition. In this section we propose to study the class $\mathscr{L}=\{A \mid A / \mathscr{D}(A)$ is injective $\}$ of modules over an arbitrary ring with unit.

In [1] the first Goldie torsion derived functor of an $R$-module $A$, which is denoted by $\operatorname{Gold}^{1}(A)$, is defined as follows:

$$
\operatorname{Gold}^{1}(A)=\frac{E(A)}{A} / \frac{E(\mathscr{D}(A))+A}{A} .
$$

From this formula it follows easily that for any $R$-module $A, A / \mathscr{D}(A)$ is injective if and only if $\operatorname{Gold}^{1}(A / \mathscr{D}(A))=0$. [1] Theorem 1.2 shows any exact sequence $0 \rightarrow A \rightarrow B \rightarrow C \rightarrow 0$ induces $0 \rightarrow \mathscr{D}(A) \rightarrow \mathscr{D}(B) \rightarrow$ $\mathscr{D}(C) \rightarrow \operatorname{Gold}^{1}(A) \rightarrow \operatorname{Gold}^{1}(B) \rightarrow \operatorname{Gold}^{1}(C) \rightarrow 0$ exact.

Proposition 3.1. (1) $\mathscr{L}=\{A|A| \mathscr{D}(A)$ is injective $\}$ is a class closed under homomorphic images, extensions, finite direct sums, and injective envelopes.

(2) $\mathscr{L}$ is a torsion class if and only if any direct sum of $\mathscr{D}$ torsionfree injectives is injective.

Proof. (1) These properties are easily verified using the results of [1].

(2) By (1), $\mathscr{L}$ is a torsion class if and only if $\mathscr{L}$ is closed under (arbitrary) direct sums. Suppose the direct sum of $\mathscr{D}$-torsionfree injectives is injective, and let $\left\{A_{\alpha}\right\}_{\alpha \in W}$ be a set of elements of $\mathscr{L}$. Then $A_{\alpha} / \mathscr{D}\left(A_{\alpha}\right)$ is injective, and so $\oplus \sum_{\alpha \in W} A_{\alpha} / \mathscr{D}\left(A_{\alpha}\right)$ is injective by hypothesis. Thus Gold ${ }^{1}\left(\oplus \sum_{\alpha \in W} A_{\alpha} / \mathscr{D}\left(A_{\alpha}\right)\right)=0$, and hence $\bigoplus \sum_{\alpha \in W} A_{\alpha} / \mathscr{D}\left(A_{\alpha}\right) \in \mathscr{L}$. Also

$$
\operatorname{Gold}^{1}\left(\oplus \sum_{\alpha \in W} \mathscr{D}\left(A_{\alpha}\right)\right)=\operatorname{Gold}^{1}\left(\mathscr{D}\left(\oplus \sum_{\alpha \in W} A_{\alpha}\right)\right)=0,
$$


and hence $\oplus \sum_{\alpha \in W} \mathscr{D}\left(A_{\alpha}\right) \in \mathscr{L}$. But the exact sequence

$$
0 \rightarrow \bigoplus \sum_{\alpha \in W} \mathscr{D}\left(A_{\alpha}\right) \rightarrow \bigoplus \sum_{\alpha \in W} A_{\alpha} \rightarrow \bigoplus \sum_{\alpha \in W} A_{\alpha} / \mathscr{D}\left(A_{\alpha}\right) \rightarrow 0
$$

induces the exact sequence

$0=\operatorname{Gold}^{1}\left(\bigoplus \sum_{\alpha \in W} \mathscr{D}\left(A_{\alpha}\right)\right) \rightarrow \operatorname{Gold}^{1}\left(\oplus \sum_{\alpha \in W} A_{\alpha}\right) \rightarrow \operatorname{Gold}^{1}\left(\oplus \sum_{\alpha \in W} A_{\alpha} / \mathscr{D}\left(A_{\alpha}\right)\right)=0$.

Hence $\oplus \sum_{\alpha \in W} A_{\alpha} \in \mathscr{L}$ by exactness.

Conversely, if $\mathscr{L}$ is closed under direct sums, then the direct sum of $\mathscr{D}$-torsionfree injectives, being both $\mathscr{D}$-torsionfree and in $\mathscr{L}$, is injective.

As in [1] we call $F \in \mathscr{F} \mathscr{D}$-absolutely pure if $A \supseteqq F$ and $A \in \mathscr{F}$ imply $A / F \in \mathscr{F}$.

Proposition 3.2. (1) If $\mathscr{D}$ is closed under products, so is $\mathscr{L}$. (2) The following are equivalent:

(i) $\mathscr{L}$ is closed under submodules.

(ii) $\mathscr{L}$ is all $R$-modules.

(iii) $R=\mathscr{D}(R)+S$ where $S$ is semisimple with minimum conditions.

(3) The following are equivalent for $A \subseteq B, B \in \mathscr{L}$ :

(i) $A \in \mathscr{L}$.

(ii) $A / \mathscr{D}(A)$ is $\mathscr{D}$-absolutely pure.

(iii) $B / A+\mathscr{D}(B)$ is $\mathscr{D}$-absolutely pure.

Proof. (1) Let $\left\{A_{\alpha} \mid \alpha \in W\right\}$ be a set of elements of $\mathscr{L}$. Then:

$\operatorname{Gold}^{1}\left(\prod_{\alpha \in W} A_{\alpha}\right)=\frac{E\left(\pi A_{\alpha}\right)}{\pi A_{\alpha}} / \frac{\left(E\left(\pi A_{\alpha}\right)\right)+\pi A_{\alpha}}{\pi A_{\alpha}}$

$$
\cong \prod_{\alpha \in W}\left[\frac{E\left(A_{\alpha}\right)}{A_{\alpha}} / \frac{\left(E\left(A_{\alpha}\right)\right)+A_{\alpha}}{A_{\alpha}}\right]=\prod_{\alpha \in W} \operatorname{Gold}^{1}\left(A_{\alpha}\right)=0 .
$$

(2) This is easily verified using [1] Theorem 3.1.

(3) ( i ) $\Rightarrow$ (ii) is immediate from [1] Proposition 1.4.

The exact sequence

$$
0 \rightarrow A / \mathscr{D}(A) \rightarrow B / \mathscr{D}(B) \rightarrow B / A+\mathscr{D}(B) \rightarrow 0
$$

induces the exact sequence

$$
\begin{gathered}
\mathscr{D}(B / \mathscr{D}(B)) \rightarrow \mathscr{D}(B / A+\mathscr{D}(B)) \rightarrow \operatorname{Gold}^{1}(A / \mathscr{D}(A)) \rightarrow \\
\operatorname{Gold}^{1}(B / \mathscr{D}(B)) \rightarrow \operatorname{Gold}^{1}(B / \mathscr{D}(B)+A) \rightarrow 0 .
\end{gathered}
$$

Since $B \in \mathscr{L}$, this sequence reduces to

$$
0 \rightarrow \mathscr{D}(B / A+\mathscr{D}(B)) \rightarrow \operatorname{Gold}^{1}(A / \mathscr{D}(A)) \rightarrow 0 \rightarrow \operatorname{Gold}^{1}(B / \mathscr{D}(B)+A) \rightarrow 0
$$


from which (ii) $\Rightarrow$ (iii) follows by [1] Proposition 1.4 .

Proposition 3.3. Let $\mathscr{L}$ be a torsion class. Then:

(1) $V=\bigcap_{F \in \mathscr{F}}(0: F)=\bigcap_{F \in \mathscr{F}}(0: E(F))$ and there exists $G \in \mathscr{F}$ such that $(0: G)=V$.

(2) $\mathscr{L}$ is the smallest torsion class containing $\mathscr{D}$ and the injective $R$-modules.

(3) The torsionfree class corresponding to $\mathscr{L}$ is $\left\{B \mid \operatorname{Gold}^{1}(C) \neq 0\right.$ for all nonzero $\left.C \leqq B\right\}$ •

Proof. (1) Let $\left\{I_{\alpha} \mid \alpha \in W\right\}$ be a set of representatives of the injective hulls of the cyclic elements of $\mathscr{F}$. Setting $G=\oplus \sum_{\alpha \in W} I$, the claim can be easily verified.

(2) Clearly, $\mathscr{L}$ contains $\mathscr{D}$ and the injective modules. If $A \in \mathscr{L}$, then it follows from the exact sequence $0 \rightarrow \mathscr{D}(A) \rightarrow A \rightarrow$ $A / \mathscr{D}(A) \rightarrow 0$ that $A$ is in the torsion class generated by $\mathscr{D}$ and the injectives.

(3) This is easily verified.

\section{REFERENCES}

1. J. S. Alin and S. E. Dickson, Goldie's torsion theory and its derived functor, (to appear in Pacific J. Math.)

2. B. Banaschewski, On coverings of modules, Math. Nachr. 31 (1965), 57-71.

3. S. E. Dickson, A torsion theory for abelian categories, Trans. Amer. Math. Soc. 121 (1966), 223-235.

4. E. Enochs, Torsionfree covering modules, Proc. Amer. Math. Soc. (1963), 884-890.

5. C. Faith and E. A. Walker, Direct sum representations of injective modules, Journal of Algebra 5, (1967), 203-221.

6. J. P. Jans, Some aspects of torsion, Pacific J. Math. 15 (1965), 1249-1259.

7. B. Stenström, Pure submodules, Arkiv for Mathematik 7 (1967), 159-171.

8. - Direct sum decompositions in Grothendiek categories (to appear)

Received August 30, 1967. This work was supported by a NASA fellowship and will compose a part of the author's doctoral thesis at the University of Nebraska.

The author wishes to express his sincere gratitude to his advisor S. E. Dickson for his help and encouragement.

UNIVERSITY OF NEBRASKA

LINCOLN, NEBRASKA 



\section{PACIFIC JOURNAL OF MATHEMATICS}

\section{EDITORS}

H. ROYDEN

Stanford University

Stanford, California

\section{R. $R$ PHELPS}

University of Washington

Seattle, Washington 98105

\section{J. DugunduI}

Department of Mathematics

University of Southern California

Los Angeles, California 90007

RICHARD ARENS

University of California

Los Angeles, California 90024

\section{ASSOCIATE EDITORS}

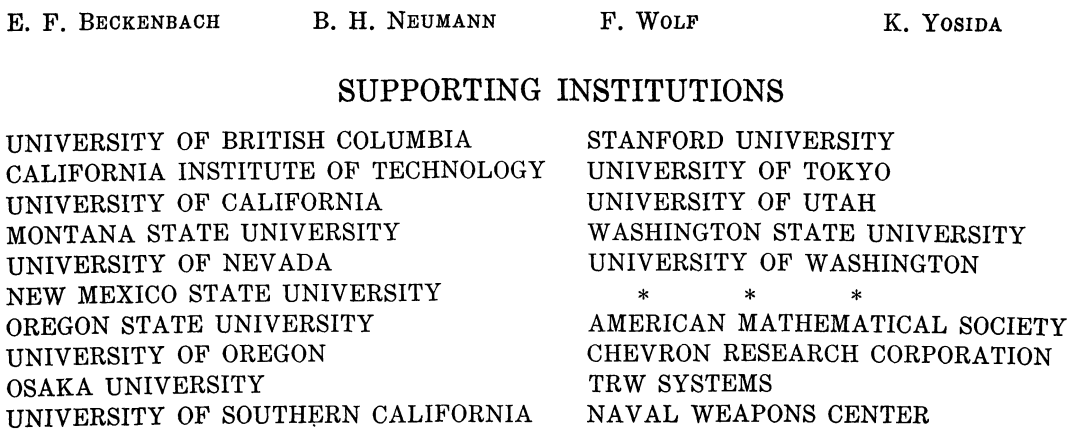

The Supporting Institutions listed above contribute to the cost of publication of this Journal, but they are not owners or publishers and have no responsibility for its content or policies.

Mathematical papers intended for publication in the Pacific Journal of Mathematics should be in typed form or offset-reproduced, double spaced with large margins. Underline Greek letters in red, German in green, and script in blue. The first paragraph or two must be capable of being used separately as a synopsis of the entire paper. It should not contain references to the bibliography. Manuscripts, in duplicate if possible, may be sent to any one of the four editors. Please classify according to the scheme of Math. Rev. 36, 1539-1546. All other communications to the editors should be addressed to the managing editor, Richard Arens, University of California, Los Angeles, California, 90024.

50 reprints are provided free for each article; additional copies may be obtained at cost in multiples of 50 .

The Pacific Journal of Mathematics is published monthly. Effective with Volume 16 the price per volume (3 numbers) is $\$ 8.00$; single issues, $\$ 3.00$. Special price for current issues to individual faculty members of supporting institutions and to individual members of the American Mathematical Society: $\$ 4.00$ per volume; single issues $\$ 1.50$. Back numbers are available.

Subscriptions, orders for back numbers, and changes of address should be sent to Pacific Journal of Mathematics, 103 Highland Boulevard, Berkeley, California, 94708.

PUBLISHED BY PACIFIC JOURNAL OF MATHEMATICS, A NON-PROFIT CORPORATION

Printed at Kokusai Bunken Insatsusha (International Academic Printing Co., Ltd.), 7-17, Fujimi 2-chome, Chiyoda-ku, Tokyo, Japan. 


\section{Pacific Journal of Mathematics}

Vol. 28, No. $2 \quad$ April, 1969

Richard Arens and Donald George Babbitt, The geometry of relativistic

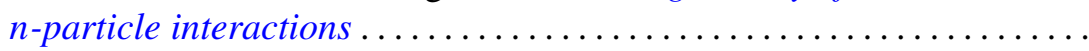

Kirby Alan Baker, Hypotopological spaces and their embeddings in lattices

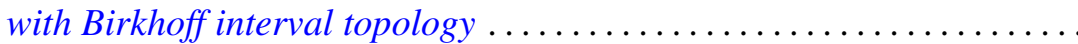

J. Lennart (John) Berggren, Finite groups in which every element is conjugate to its inverse ........................... 289

Beverly L. Brechner, Homeomorphism groups of dendrons . . . . . . . . . . . 295

Robert Ray Colby and Edgar Andrews Rutter, QF - 3 rings with zero singular ideal ................................. 303

Stephen Daniel Comer, Classes without the amalgamation property....... 309

Stephen D. Fisher, Bounded approximation by rational functions ......... 319

Robert Gaines, Continuous dependence for two-point boundary value problems..................................... 327

Bernard Russel Gelbaum, Banach algebra bundles ............... 337

Moses Glasner and Richard Emanuel Katz, Function-theoretic degeneracy criteria for Riemannian manifolds ...................... 351

Fletcher Gross, Fixed-point-free operator groups of order $8 \ldots \ldots \ldots \ldots 357$

Sav Roman Harasymiv, On approximation by dilations of distributions . . . . 363

Cheong Seng Hoo, Nilpotency class of a map and Stasheff's criterion ... . . 375

Richard Emanuel Katz, A note on extremal length and modutus.......... 381

H. L. Krall and I. M. Sheffer, Difference equations for some orthogonal polynomials ................................

Yu-Lee Lee, On the construction of lower radical properties ........... 393

Robert Phillips, Liouville's theorem........................... 397

Yum-Tong Siu, Analytic sheaf cohomology groups of dimension $n$ of

n-dimensional noncompact complex manifolds ..... . .

Michael Samuel Skaff, Vector valued Orlicz spaces. II...

James DeWitt Stein, Homomorphisms of $B^{*}$-algebras .... . .

Mark Lawrence Teply, Torsionfree injective modules .... . . .

Richard R. Tucker, The $\delta^{2}$-process and related topics. II .

David William Walkup and Roger Jean-Baptiste Robert Wets, Lifting

projections of convex polyhedra...

Thomas Paul Whaley, Large sublattices of a lattice. 